\title{
On CFD and transient flow in vehicle aerodynamics
}

\author{
Sven Perzon \\ Volvo Car Corporation \\ Lars Davidson \\ Chalmers University of Technology
}

Copyright @ 1998 Society of Automotive Engineers, Inc.

\begin{abstract}
The accuracy of computational fluid dynamics, CFD, has improved considerably over the years but still, large errors are present and vehicle parameters such as drag and lift are often poorly predicted.
\end{abstract}

The current wor $k$ is inv estigating how $\operatorname{tr}$ ansient CF D would cope with a v ery complex flow s tructure around a surface mounted c ube. A tr ansient Rey nolds av eraged Navier Stokes model, RANS model, is presented together with a lar ge eddy simulation model, LES model.Furthermore, two "industrial like" test cases have been simulated using a transient RANS model.

\section{INTRODUCTION}

Today CF D is us ed in the dev elopment of new trucks and cars at Volvo. Typically a new $\mathrm{c}$ ar or truck design is evaluated within 5-10 day $s$ after receiving a CAD database. Block-structured meshes of 2-4 million c ells are generated with ICEM CFD. The equations are solved with the StarCD flow s olver from Computational Dynamics Ltd. CFD is used to compare different design proposals, to do basic shape studies in the early stages of a project and to do s hape optimization in later stages. The method pr edicts tr ends in dr ag with a $v$ ery high accuracy in most cases but sometimes still fails to do so. If CFD should be used on its own without model tests, the accuracy $r$ equirement inc reases and $d r$ ag and lift must be truthfully simulated in the future.

In previous work by Ramnefors et. al. (1996), the base pressure error was believed to originate from an erroneous pr ediction of wher e the s eparation line was located. This is a fact if the geometry does not have a separation line that is well defined. Howev er, studies of the flow around a s implified tr uck with a $s$ harp tr ailing edge showed that ev en though the separation is geometrically well defined, similar error levels in the base pressure occur, see Perzon, Sjögren \& Jönson (1998). This indicates that an er roneous predicting of the wak $e$ structure causes the error.

The mes h has been one of the major issues in all the accuracy work done at Volv $\mathrm{o}$. It is shown by Ramnefors et. al. (1996) and Ax elsson et. al. ( 1998) that mes $h$ quality may be jus $t$ as impor tant as mesh resolution when accuracy is searched for.

Different turbulence models have been used for the timeaveraged flow. Some turbulence models mimic the wake structure well and others do not. Reynolds Stress Models have been used in the simulation of the flow around the Volvo ECC, ( Ramnefors et al. 1996). It was shown that the qualitative pressure distribution was more accurately described but the base pressure level is poorly predicted. However, these complex models are often numerically troublesome, Perzon (1997). Hence, the route has been taken towards non-linear eddy $\mathrm{v}$ iscosity models and algebraic Reynolds stress models see Perzon, Sjögren \& Jönson (1998).

Numerical problems often occur when the mesh resolution becomes very fine around regions where a separation is present, e.g. the tr ailing edge. $T$ he separation line tends to move around when the s olution is sought for during iterations and a fully converged solution may not be pos sible to find. If the geometr $y$ is complex enough s o that poor mes $h$ quality is unavoidable and/or if a complex turbulence model is used, the convergence problem will get even worse. It is often a fact that the wak $e$ is not stationary and the attempt to solve the problem based on that as sumption, cease to work at some modeling point. E.g. when the accuracy in the model is large enough to discover this fact. The obvious solution to the convergence problem is to solve the equations us ing a tr ansient s olution methodology and this is what the present work will describe. 
Transient simulations around vehicles have been presented previously by Summa (1992) and it was used for testing the vehicle for reactions of a pitch oscillation. The simulation was for demonstrational purposes and it shows an ex ample of how tr ansient simulations may be applied in a car project. Another ar ea of inter est is aerodynamical for ces of $a v$ ehicle in cross wind gusts. Within that area only measurements have been presented previously, see Garry et. al. (1994) and the work by Ryan and Dominy (1998). This would be an excellent topic in the futur e for transient CFD. It should be noted also that Hucho and Emmelmann (1974) have proposed a theoretical prediction method.

Another area where transient CFD would be applicable is passenger comfort in the tail coach of a train, which is known to be affected so that it vibrates slowly sideways by the time dependent side force caused by the transient wake. The latter is closely related to the current topic.

Tsuboi et. al. (1988) and Kataoka et. al. (1991) presented early transient simulations using quasi-direct numerical simulations, DNS. It is a fact that for such high Reynolds number, there is not a computer for sale today that could solve a tr ue DNS of the flow around a car. Hence, when the transient Navier Stokes equations are solved without any modeling, it should be called DNS but since the r esolution is way too $c$ oarse, I hav e preferred to call it, quas i-DNS. In T suboi et. al. (1988) the flow around a body with a slanted rear was simulated and the critical angle $\mathrm{c}$ ould be detec ted in the simulations. In
Kataoka et. al. (1991), comparisons with forces and surface pressures fr om ex periments agr eed $v$ ery well even for add on detailing. Yamada and Ito (1993) made a comparison between a tr ansient quas i-DNS, and a stationary simulation using the $\mathrm{k}-\varepsilon$ model. It was shown that the stationary model was able to pr edict the flow field qualitatively $\mathrm{c}$ orrect but that it failed with the simulation of ac curate for ces. The quasi-DNS method simulated the $s$ urface pr essure dis tribution poor ly but predicted the forces fairly accurate. No data was presented on the transient behavior of the wake. The fact that forces may be accurately simulated for all the wrong reasons is something that is fairly common, see Perzon et. al. ( 1998). F urthermore, Hor inouchi et. al. ( 1995), presented some results from a quasi DNS simulation where embedded grids was used. Also in this case, only stationary data was presented and the method was able to produce very ac curate tr ends on aer odynamical forces. Aoki et. al. (1993) presented simulations on some test $c$ ases us ing a lar ge eddy $s$ imulation, LES. The subgrid s cale model pr esented was a Smagorinsky (1965) model and the trends in drag were fairly accurately predicted. The critical r oof slope is found in the simulations and some transient data is shown.

Duell and George (1992) did transient measurements on a three dimensional bluff body in pr oximity to a gr ound plane with and without a splitter plate in the near wake. The measurement indicates that the aerodynamic drag is coupled to transient effects in the wake.

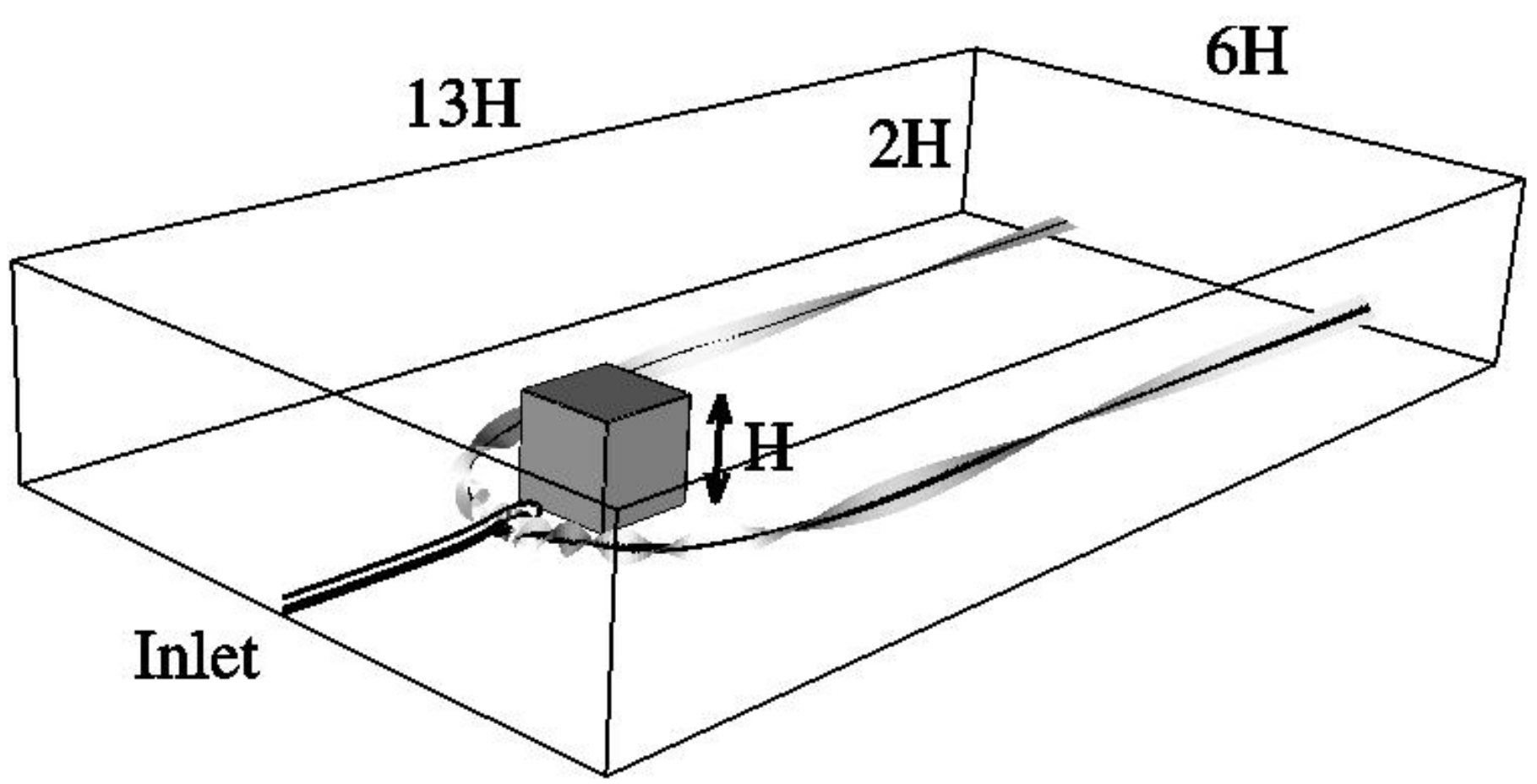

Figure 1 Computational domain and visualisation of the horse shoe vortex with streamlines from the averaged velocity field of the LES simulation. 
Unsteady pressure measurements in the wake have been done recently by Ishihara and Takagi (1999) where total pressure was meas ured at frequencies up to 300 $\mathrm{Hz}$ with and without moving belt. Comparisons were made between mean and instantaneous data and the difference were large implying that the wak e large-scale movement is considerable for that vehicle.

\section{SURFACE MOUNTED CUBE}

The surface mounted cube has been measured by Martinuzzi and Tropea, 1993, and was used as the main test c ase for this study. Sev eral other s have studied it before, e.g. see Krajnovic and Davidson (1999). Furthermore it has been one of the test cases in the ERCOFTAC "classic collection" database, which means that the measurements hav e been $c$ hecked by an independent academic community which give reason to believe that it is measurements of high quality. Database adress:

\section{http://vortex.mech.surrey.ac.uk/}

\section{THE COMPUTATIONAL SETUP}

The computational domain was created with the ability of testing s everal models and s chemes and henc e, the model had to be kept fairly coarse.

\section{The mesh}

ICEM Mulcad was used for grid generation and the mesh that was us edfor the s tudy had the following characteristics,

- Number of cells: 264576 hexahedral cells.

\section{- Block structured grid}

- The simple geometry made it possible to obtain perfect orthogonality everywhere in the mesh

The inlet of the domain is located 3 cube heights upstream of the cube and the overall length of the domain is 13 cube heights, see figure 1 .

\section{Turbulence models}

In Hucho (1998), CFD methods are classified into several groups where the latter three groups are,

- Time averaged, steady, RANS methods

- Large eddy simulation, LES

- Direct numerical simulation, DNS

Of thes e groups the fir st s tationary RANS is the most widely used and mostly in conjunction with the $k-\varepsilon$ turbulence model. The reasons for using the $\mathrm{k}-\varepsilon$ model are that the model is numerically robust and that is has been used for quite some time, giving presence to an extensive validation database. The latter $r$ eason if of great importance in industrial applications. A tremendous amount of work would be $r$ equired to build a s imilar knowledge about another model and hence the k$\varepsilon$ model is used even though it is known to give the wrong ans wer in $\mathrm{m}$ any ty pical situations. Stagnation regions and regions with high streamline curvature are typical flows $w$ here the $m$ odel $f$ ails. Due to the historic rucksack it is troublesome for industry to start using more elaborate turbulence models since many previous simulations have to be $r$ ecomputed with the new model. A similar problem about pas senger $\mathrm{c}$ omfort $\mathrm{w}$ ithin the aircraft industry is nicely pin pointed in Adams (1997).

As des cribed below, RANS $c$ an be used in transient simulations. Since LES, is s till too ex pensive in mos $t$ cases, tr ansient RANS may be an alter native for some years until c omputers are fast enough to allow for LES simulations.

As hinted previously, two different classes of tur bulence models have been used during the course of this study and these are a time av eraging method, RANS, and a method that is filtering the equations of state, LES.

The time averaging approach decomposes velocities and pressure into a mean and fluctuating part and the Navier Stokes equations ar e time av eraged over some time interval, T, and the Reynolds equations appear,

$$
\frac{D U_{i}}{D t}=-\frac{1}{\rho} \frac{\partial P}{\partial x_{i}}+\frac{\partial}{\partial x_{j}}\left(\nu \frac{\partial U_{i}}{\partial x_{j}}-\overline{u_{i} u_{j}}\right)
$$

A new term has to be tak en $c$ are of by $s$ ome $k$ ind of turbulence model. As stated above, the most common choice in indus trial applic ations is the $s$ tandard $k-\varepsilon$ model, Jones and Launder (1972). In this study a more advanced two-equation model hav e been used and it is the quadratic stress-strain relationship proposed by Shih et. al. (1991), her eafter denoted the SZL-model. Even though a time averaging has been imposed to the equations, a transient simulation $c$ an be appr opriate if the time averaging interval is long enough to embed the turbulent time $\mathrm{s}$ cales and $\mathrm{s}$ hort enough to $\mathrm{c}$ apture the large scale mean flow movements. In a flow with a moving boundary such as an in-cylinder flow it is easily understood that some scales are turbulent and that some scales originate from moving boundaries, e.g. piston and valves. In aerodynamics the boundaries are stationary and it may be har der to separate turbulence from mean flow variations. One may suspect that such a model would work properly only when a clear scale separation is present. An example of such a case would be an obvious v ortex s hedding behind a bluff body. Then the mean flow variations would be the moving boundary of the wake while the $c$ haotic s tructure ins ide the wake 
would be turbulence. If the wake movement would have several characteristic frequencies and the s cales spans from the largest scales to the smallest turbulent scales, it will be tricky to choose the time averaging period, $\mathbf{T}$, that fulfilled the requirement described previously. The transient approach using a tur bulence model is us ually called T RANS, ( Transient Rey nolds Averaged Navier Stokes).

The other approach that has been used is a large eddy simulation, LES, s trategy. Dir ect simulation of all scales in a turbulent flow is feasible only at very low Reynolds numbers. This suggests a simulation technique based on some decomposition of the flow into lar ge and $s$ mallscale structures. $T$ he lar ge s cales ar e then dir ectly solved for in thr ee-dimensional time-dependent fashion and the small scales ar e modeled. $T$ his appr oach is called LES, ( Large Eddy Simulation). The major reason for testing such a model in this work is that it should, by definition, work better in flows where there is a lac $k$ of scale separation since the model is des igned to tak $e$ care of all lar ge tr ansient s cales, tur bulent or not. T he velocities and the pressure are decomposed into a resolved part (still transient) and a subgrid part, which is modeled. The result becomes similar to the Reynolds equations,

$$
\frac{D \bar{\pi}_{i}}{D t}=-\frac{1}{\rho} \frac{\partial \bar{p}}{\partial x_{i}}+\frac{\partial}{\partial x_{j}}\left(\nu \frac{\partial \bar{\pi}_{i}}{\partial x_{j}}-\tau_{i j}\right)
$$

The last term have to be modeled and the most simple sub-grid model available is the S magorinsky (1965) and it has been used in this study. The grid length scale was chosen as, $\mathrm{V}^{1 / 3}$, where $\mathrm{V}$ is the grid cell volume. A nice overview of other interesting sub-grid models tes ted on flows around bluff bodies can be found in Murakami (1998).

The subgrid model in the LES model will vanish when the mesh is refined to an extent that all fine scales are resolved. Thus, the LES model becomes a direct numerical simulation when the mesh and time step allows for it. The turbulence model in the T RANS model will never fade out because of a fine mesh resolution.

\section{Boundary conditions}

The inlet boundary was taken from experiments, Martinuzzi and Tropea, 1993. This experimental database also provided data for the nor mal and $s$ hear stresses at the inlet and the tur bulence properties $\mathrm{k}$ and $\varepsilon$ were set, if appropriate, according to,

$$
\varepsilon=\frac{k^{2}}{C_{\mu}{ }^{-0.75} \max (k \eta, \lambda \delta)}
$$

where $\eta$ is the distance to the wall, $\delta$ is the boundary layer thickness at the inlet and $\lambda=0.09$ for channel flows.

At the walls the wall function approach was used on the tunnel roof, on the cube and on the tunnel floor. The sides of the domain were treated as slip walls, which also was used in the work of Krajnovic and Davidson (1999).

\section{Numerical model}

The commercial solver StarCD was used throughout this study and the version was 3.05. T he RANS model is available in the $c$ ode and the LES model was eas ily incorporated via user coding.

The Crank Nicholson scheme was used for the time discretization. $T$ wo differ ent $s$ patial dis cretization schemes were tes ted and thes e ar e the $c$ entral differencing scheme, which is $2^{\text {nd }}$ order accurate, and the MARS scheme, which also, is a $2^{\text {nd }}$ order accurate scheme. Normally the c entral differ encing, CD, scheme is not robust enough in stationary simulations but since the simulation is time dependent, it s eems to be robust enough and a very accurate and "low cost" scheme to use. The MARS scheme with two different blending factors, 0.5 and 1.0, that controls the amount of upwinding in the scheme. Low values of this factor introduces more upwinding. The MARS scheme is second order accurate regardless of the blending factor.

The equations are solved for using the PISO algorithm. Different time steps have been tested for the transient RANS model but in all comparisons between the transient RANS and the LES model, an identical time step was us ed, $\Delta \mathbf{t}=\mathbf{0 . 0 1} \mathrm{s}$, which corresponds to a maximum Courant number around 3 .

\section{RESULTS}

The initial s imulation that hav e to be per formed prior to any data $c$ ollection was $s$ et to ten time scales, $\tau$. The time scale, $\tau$, is the time it takes for a fluid particle in the free-stream to pass the computational domain, e.g. $\tau=10$ s. Hence, with $\Delta \mathbf{t}=\mathbf{0 . 0 1} \mathbf{s}$ it takes 100.000 time steps before data collection can be started.

$$
k=\frac{1}{3} \overline{u_{i} u_{i}}
$$




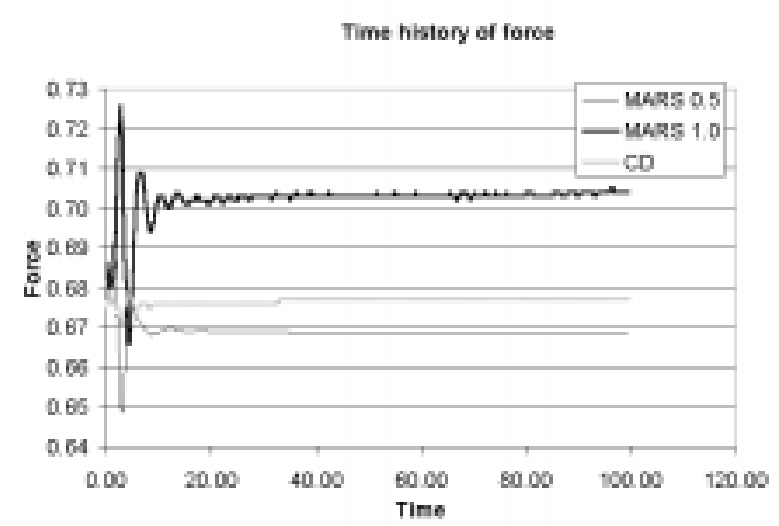

Figure 2. Time history of the force on the surface mounted cube during the initialization phase. A comparison between different advection schemes. $\Delta t=0.1$ seconds.

The aerodynamical drag force of the cube is shown in figure 2 as a function of time during the initialization phase for the SZL model. The effects of the chosen scheme is shown and as $c$ an be $s$ een the $v$ ery simple $\mathrm{CD}$ scheme is similar to the muc $\mathrm{h}$ more elabor ate and computationally expensive MARS scheme. In figure 3, a similar plot is showing the effect caused by different time steps using the SZL model. If the time s tep is chosen as too coarse, it may be a fact that transient behavior will be damped out. It is also shown that the drag seems to have just about s ettled and the initializ ation phase appears to be long enough. The averaging are done from the end of this initializ ation phase and the $s$ tatistics was collected for another 10 time scales described above.

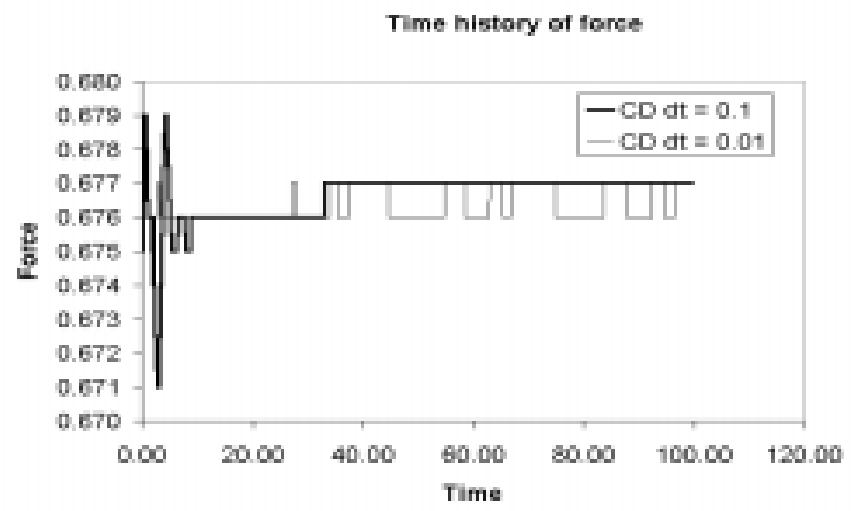

Figure 3. Time history of the force on the surface mounted cube during the initialization phase. A comparison between different time steps. $\Delta t=0.1$ seconds corresponds to a maximum Courant number around 30 and $\Delta \mathrm{t}=0.01$ seconds coincide with ditto around 3.

In figure 4 comparisons are made between LES, transient RANS and ex periments, $0.5 \mathrm{c}$ ube heights upstream of the front face on the cube, see figure 1. It is readily shown that the transient RANS model is fairly close to ex periment. $T$ he ex perimental pos ition goes through the hor se s hoe $v$ ortex and the RANS model mimics that quite well. It exaggerates the back-flow somewhat. The LES model s eems to have a problem at $\mathrm{Y} / \mathrm{H}=1.1$ where an unphy sical peak is shown when the nondiffusive central differencing scheme, CD scheme, is used. The same turbulence model does not show this when the MARS scheme is us ed. The MARS scheme and the CD scheme are both second order accurate but the MARS scheme c ontains s ome upwinding which in this case damps out the numer ical ins tabilities that causes the peak whereas the CD s cheme doesn't. It is interesting to note that the TRANS model does not show this behaviour even though the CD s cheme is used. The turbulence model incorporates enough turbulent diffusion to damp out this phenomenon. T he LES model exaggerates the bac kflow $s$ omewhat less than the TRANS model. $F$ igure $5 \mathrm{~s}$ hows the vertical velocity component at the same spot and the T RANS model is less poor in comparison with the LES model. However, none of the models predicts the profile well in this region. In experiments the horseshoe vortex has its center closer to the $c$ ube than the X loc ation of the pr ofile. Hence, the vertical component of the velocity becomes positive close to the wall. This is not a fact in any of the simulations wher e all models have a negative vertical velocity c omponent $c$ lose to the floor imply ing that the vortex is located on the other $s$ ide of the meas uring location.

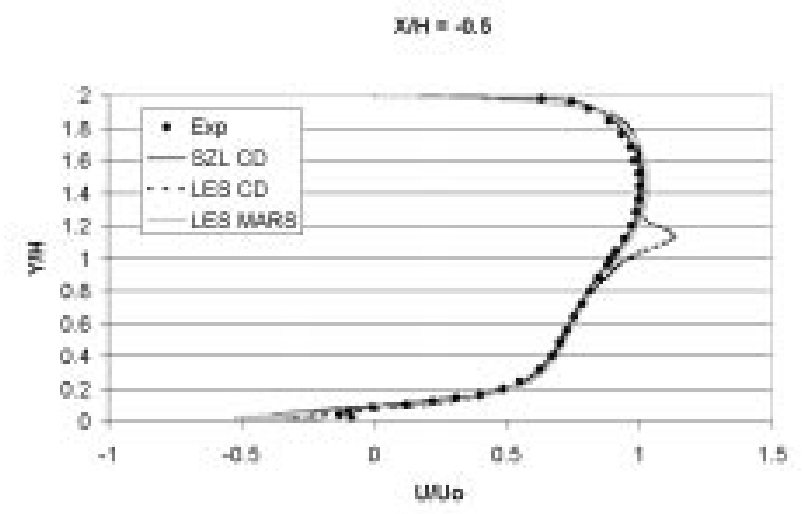

Figure 4. Streamwise velocity component, $U$, at 0.5 cube heights upstream of the cube front side, e.g. at $\mathrm{X} / \mathrm{H}=-0.5$. The data is taken along the vertical coordinate axis, $\mathrm{Y}$, in the symmetry plane, $\mathrm{Z}=0$. 


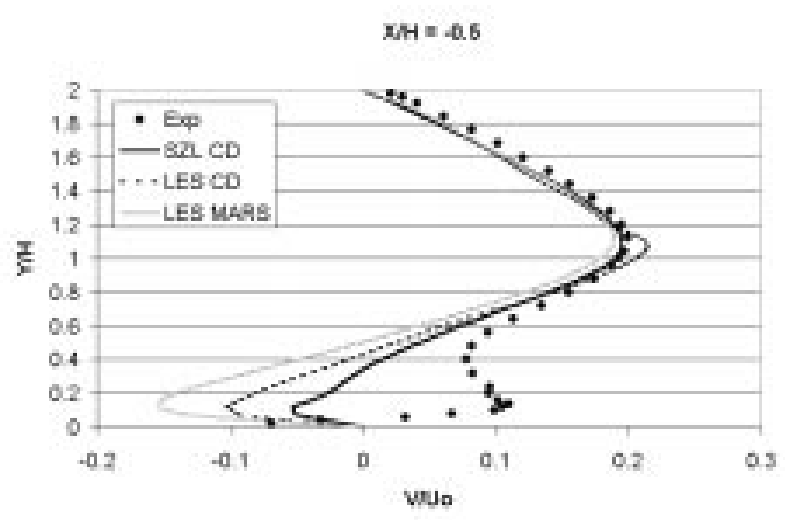

Figure 5. Vertical velocity component, V, at 0.5 cube heights upstream of the cube front side, e.g. at $\mathrm{X} / \mathrm{H}=-0.5$.

On the cube roof in figure 6 the flow fr om the front edge is shown from the cube roof to the channel roof. The TRANS model is more accurate than the LES model with the central differencing s cheme and $s$ imilar to the LES model with the MARS scheme. The next location, see figure 7 , is on the trailing edge of the cube roof. The LES model with the CD scheme underpredicts the separation on the roof and the other two models overpredicts the region of separation slightly.

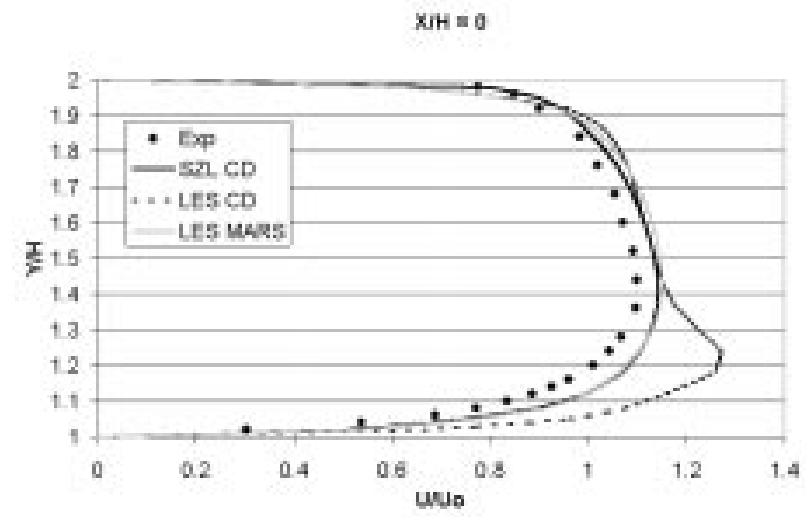

Figure 6. Streamwise velocity, U, profile on the symmetry plane and on the cube roof at $\mathrm{X} / \mathrm{H}=0$.

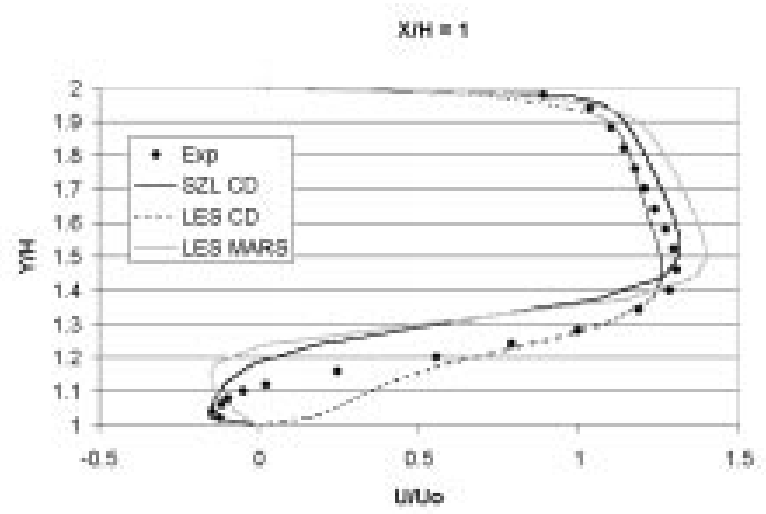

Figure 7. Streamwise velocity, U, profile on the symmetry plane and on the cube roof at the trailing edge, e.g. $\mathrm{X} / \mathrm{H}=1$.
One cube height downs tream, $\mathrm{X} / \mathrm{H}=2$, the LES MARS and the TRANS models still overdo the separation which is a direct consequence of the upstream error, see figure 8. Next location is located at $\mathrm{X} / \mathrm{H}=6$ and both the LES models closes the wake much more rapidly than the TRANS model and thus at this point, the LES models are closer to experiment then the T RANS model, s ee figure 9.

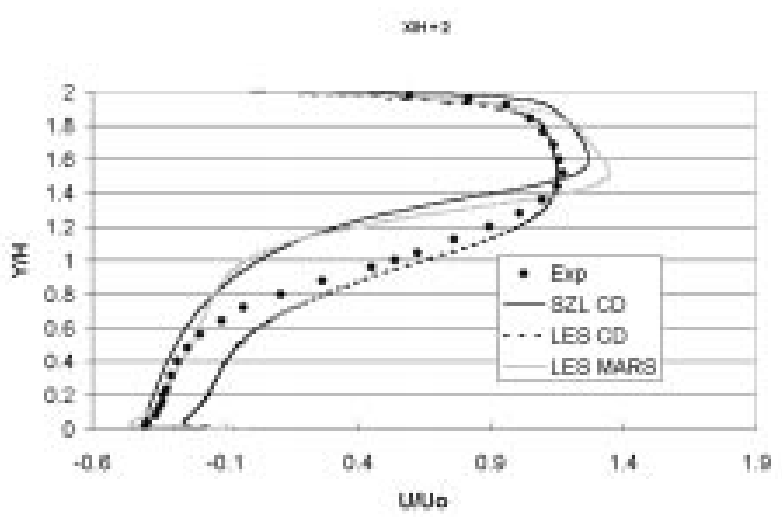

Figure 8. Streamwise velocity component, U, 1 cube height downstream of the cube trailing edge, e.g. at $\mathrm{X} / \mathrm{H}=2$.

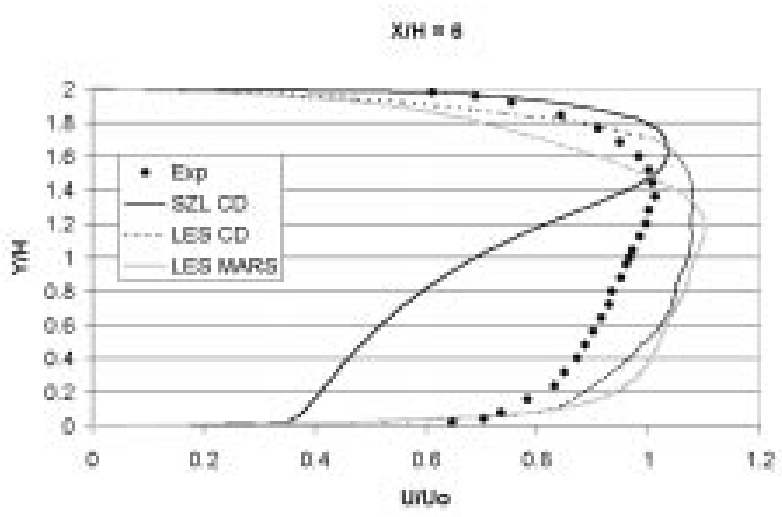

Figure 9. Streamwise velocity component, U, 5 cube height downstream of the cube trailing edge, e.g. at $\mathrm{X} / \mathrm{H}=6$.

\section{OTHER CONFIGURATIONS}

The surface mounted cube is a relevant test case in the sense that it is a well- measured test case and it has a very c omplex flow $s$ tructure. The fac $t$ that it is a very simple geometry makes it ev en more suitable for testing computational models. However, the Re number is very low, $R e=80000$, and it lacks underbody flow and hence, some other models mus t be tes ted in conjunction with the surface mounted $c$ ube. The Volv o ECC has been studied before by Ramnefor s et. al. ( 1996). The model was simulated using the same quadratic $k-\varepsilon$ model as was presented previously as the SZL-model.

The time history of the drag is shown in figure 10 . The transient behav ior $s$ ettles to a nice and smooth 
sinusoidal $\mathrm{c}$ urve. $\mathrm{T}$ he magnitude of the os cillations is however very small. It indicates that the flow behind the Volvo ECC is very calm. No large vortex structures or other "bad" aer odynamical features ex ist on that model which is shown in the very low experimental drag figure, see table 1.

\begin{tabular}{|l|l|}
\hline Configuration & $\mathrm{C}_{\mathrm{d}}$ \\
\hline Time averaged TRANS. SZL model. & 0.115 \\
\hline Stationary RANS. SZL model. & 0.111 \\
\hline Stationary RANS. k- $\varepsilon$ model. & 0.143 \\
\hline Experiments & 0.141 \\
\hline
\end{tabular}

Table 1. A comparison of drag figures between stationary and transient RANS simulations.

Stationary simulations on the identic al mes $h$ have been computed and all drag figures are tabulated in table 1. Note that the simple $k-\varepsilon$ model computes a drag figure that is very close to the meas ured value. However, the reason for this is that the model overpredicts the stagnation pressure whic h c ancel out the er ror in the base pressure. The base pressure error are of the same order for all turbulence models.

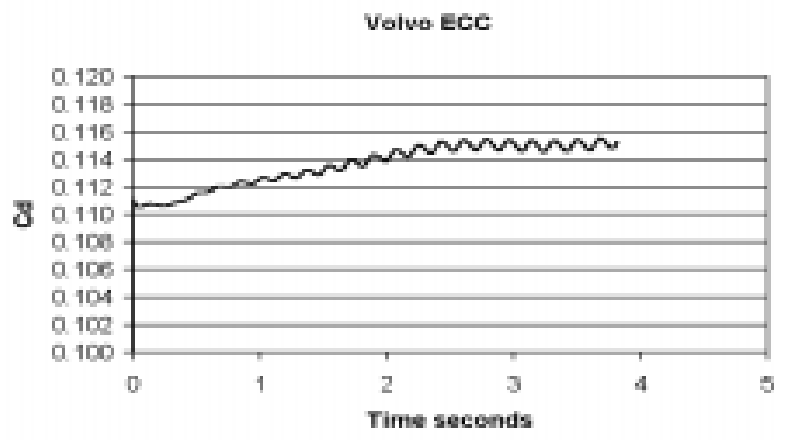

Figure 10. Time history of drag for the Volvo ECC.

A simplified Volvo FH truck have been simulated and this configuration have a very clear oscillating wake like a fish tail. The wake oscillates sideways in the simulation which is clearly shown in figure 11.

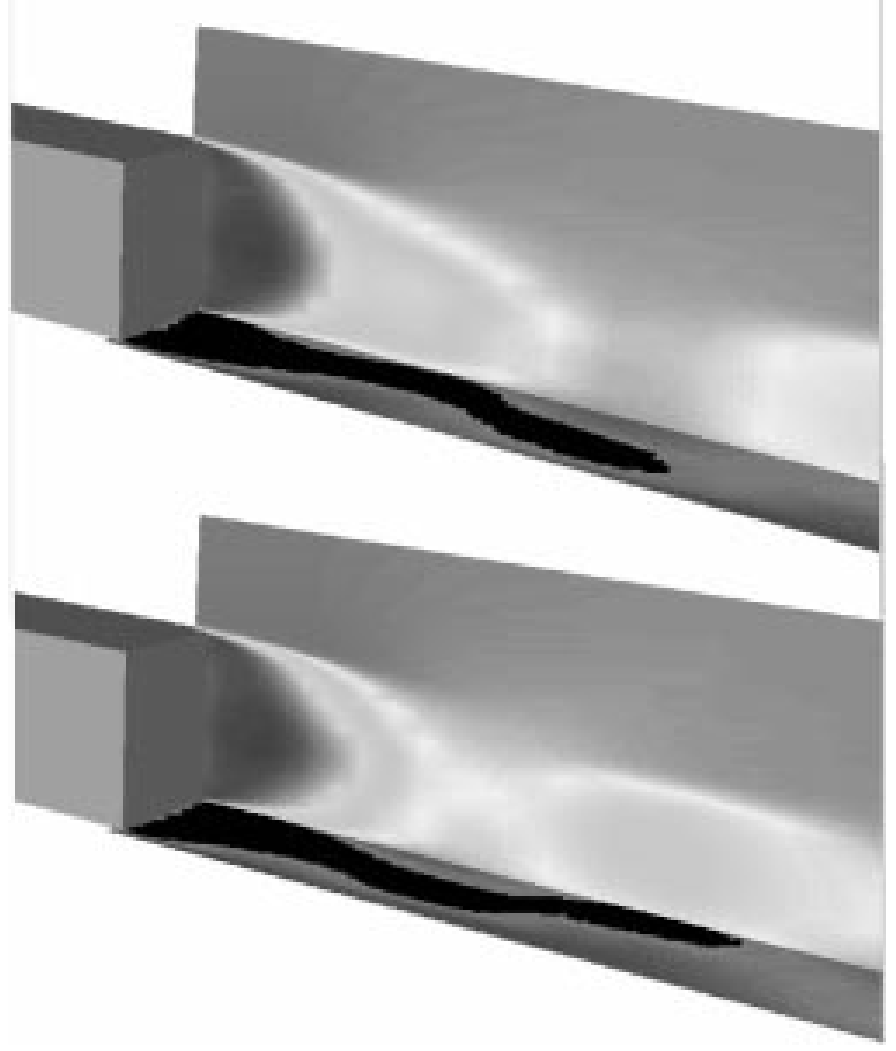

Figure 11. Velocity isocontours in the wake. Snapshots at two instants in time.

\section{CONCLUSION}

Simulation of transient flows is a fruitful path in future development of cars. The main benefits are,

- $\quad$ Stability, there is always a s olution to the equations. Hence, it doesn't matter how transient the flow is, there is always a solution.

- Physics, by solving the transient equations, more physics are imported to the problem. There is one less assumption made.

The s imulation times bec omes lar ger, around 10 times larger but the enhanced stability makes it possible to use more advanced physical models which in turn may give higher accuracy with a coarser model.

\section{CONTACT}

Sven Perzon, Volvo Car Corporation, Dept. 98365, Bld PVIB3, 40508 Göteborg, Sweden

Phone: (+46) 313213149

Fax: $\quad(+46) 31593514$

email: Sven.Perzon@hq.vcc.volvo.se 


\section{REFERENCES}

1. Adams S., The Dilbert future. Thriving on stupidity in the $21^{\text {st }}$ century., $1^{\text {st }}$ edition, HarperCollins Publishers Inc., ISBN 0-88730-866-X, 1997

2. Aoki K., Ohbayashi T., Zhu M. and Miyata H. (1993), Finite-volume simulation of 3-D vortical flow-fields about road vehicles with various after-body configuration, , SAE technical paper 931896

3. Axelsson, N., Ramnefors, M. and Gustafson, R. (1998) Accuracy in Computational Aerodynamics, Part 1: Stagnation Pressure, SAE Technical Paper 980037.

4. Duell E. G. and George A. R. (1992), Unsteady wakes of three dimensional bodies, In Bluff-body wakes, dynamics and instabilities, Eds. Eckelmann et. al., Springer-Verlag, 1992 , pp. 293-296

5. Garry K. P., Macklin A. R. and van Opstal E. P. E. (1994), Measurement of transient aerodynamic loads on bluff bodies at extreme yaw angles, In Vehicle aerodynamics, The Royal Aeronautical society, Loughborough Univ. Of Tech., UK, July 18-19, 1994

6. Horinouchi N., Kato Y., Shinano S., Kondoh T. and Tagayashi Y. (1995), Numerical investigation of vehicle aerodynamics with overlaid grid system, , SAE technical paper 950628

7. Hucho W.-H. (1998), Aerodynamics of road vehicles, $4^{\text {th }}$ edition, ISBN 0-7680-0029-7, SAE

8. Ishihara Y. and Takagi M. (1999), Unsteady pressure analysis of the wake flow behind a passanger car model, SAE technical paper 990810

9. Jones, W. P. and Launder, B. E. (1972) The prediction of laminarization with a two-equation model of turbulence, Int. J. of Heat and Mass Transfer 15, 310-314

10. Kataoka T., China H., Nakagawa K., Yanagimoto K. and Yoshida M. (1991), Numerical simulation of road vehicle aerodynamics and effect of aerodynamic devices, SAE technical paper 910597

11. Krajnovic S. and Davidson L. (1999) Large eddy simulation of the flow around a surface mounted cube using a one-equation subgrid model, In the first international symp. on turbulence and shear flow phenomena, Santa Barbara, Sept 12-15 1999

12. Martinuzzi R. And Tropea C., (1993), The Flow Around a Surface-Mounted, Prismatic Obstacles Placed in a Fully Developed Channel Flow, J. Fluids Eng. pp. 85-91, Vol. 115, March.

13. Murakami S. (1998) Overview of turbulence models applied in CWE-1997, J. of Wind Eng. and Industrial Aerodynamics, 74-76, pp. 1-24

14. Perzon, S. (1997) Reynolds Stress Modeling of Flow Separation on Curved Surfaces, Thesis for the degree of Licenciate of Engineering, Chalmers University of Technology, Göteborg, Sweden

15. Perzon, S., Sjögren, T., and Jönson, A., (1998) Accuracy in Computational Aerodynamics, Part 2: Base pressure SAE Technical Paper 980038.

16. Perzon S., Janson J. and Höglin L., (1999), On comparisons between CFD methods and wind tunnel tests on a bluff body, SAE technical paper 990805

17. Ramnefors, M., Bensryd, R., Holmberg, E., and Perzon, S. (1996) Accuracy of Drag Predictions on Cars Using CFD - Effect of Grid Refinement and Turbulence Models , SAE Technical Paper 960681.

18. Shih, T. H., Zhu, J. and Lumley, J. L. (1993) A realizable Reynolds stress algebraic equation model, NASA tech. Memo. 105993
19. Smagorinsky J., Manabe S. and Halloway J. L. (1965), Numerical results from a nine-level general circulation model of the atmosphere, Monthly weather review, 93, Dec 1965, pp. 727-768

20. Summa J. M. (1992), Steady and unsteady computational aerodynamics simulations of the Corvette ZR-1, SAE technical paper 921092

21. Tsuboi K., Shirayama S. Oana M. and Kuwahara K. (1988), Computational study of the effect of base slant, In $2^{\text {nd }}$ International Conference on supercomputing in the automotive industry, Seville Spain

22. Yamada A. and Ito S. (1993), Computational analysis of flow around a simplified vehicle-like body, , SAE technical paper 930293 